\title{
Die Behandlung einer Densfraktur
}

\author{
Michael Nerlich
}

Ein 45-jähriger Mann kommt zu Fuß in die zentrale Notaufnahme der Klinik und klagt über Kopfschmerzen. Er hatte 4 Tage zuvor einen Sturz, wo er sich heftig den Kopf angeschlagen hatte und seither Kopf- und Nackenschmerzen, die sich auch durch einen ausgiebigen, sich an das Unfallereignis anschließenden Kneipenbesuch nicht wesentlich bessern ließen.

Es wird von dem Patienten eine Röntgenuntersuchung der Halswirbelsäule durchgeführt, auf der eine instabile transdentale Luxationsfraktur festzustellen ist (Abb.1). Daraufhin erfolgt die sofortige Ruhigstellung mit einer CampKrawatte und die stationäre Aufnahme des Patienten zum Zwecke der geplanten Densverschraubung, die für den Montag der kommenden Woche terminiert wird. Der Patient selbst ist in regelrechtem Allgemeinzustand, keinerlei neurologische Ausfälle, die Kommunikation ist etwas erschwert, da er ein ausgeprägtes Stottern aufweist.

Am Wochenende klagt der Patient über vermehrte Beschwerden bei der Visite eine Röntgenkontrolle zeigt eine erneute Dislokation der Densfraktur, weshalb sich der diensthabende unfallchirurgische Oberarzt (der Autor) zu einer notfallmäßigen OP-Indikation veranlasst sieht. Die geplante Densverschraubung erfolgt daher im Wochenenddienst, intraoperativ ist die Visualisierung der Densspitze in beiden Ebenen lagerungsbedingt erschwert. Die Verschraubung mit 2 Kleinfragment-Spongiosaschrauben wird durchgeführt. Postoperativ wird der Patient in der Camp-Krawatte ruhig gestellt, er weist keine neurologischen Auffälligkeiten auf. Die postoperative Röntgenaufnahme zeigt zum einen, dass die Schraubenspitzen die Dens-

OP-JOURNAL 2008; 24: 194-195

(c) Georg Thieme Verlag KG Stuttgart • New York DOI 10.1055/s-2008-1039145
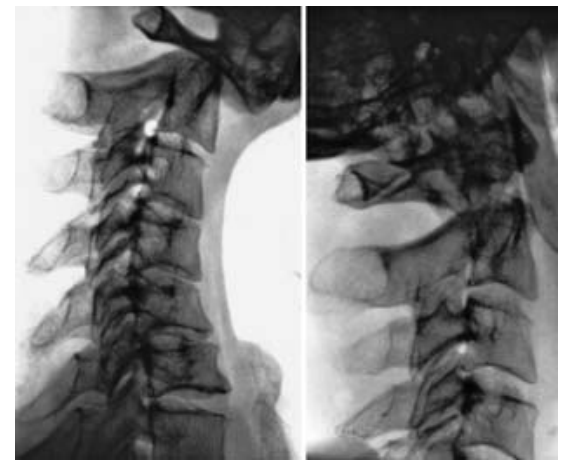

Abb. 1 Unfallbild.

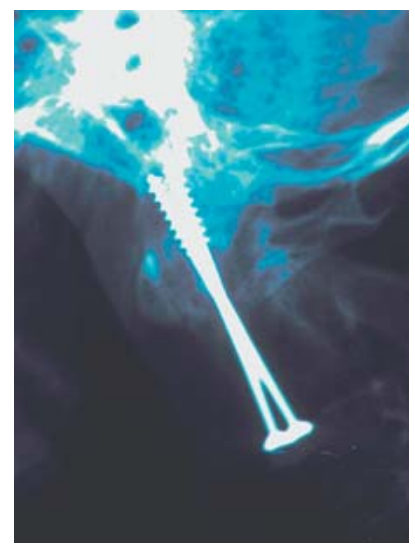

Abb. 2 Densverschraubung.

spitze überragen und zum anderen, dass die Schraubenbasis aus dem HWK2-Corpus distal ausgebrochen ist (Abb. 2).

Man entschließt sich, die Osteosynthese zu revidieren und nach Entfernung der beiden Schrauben durch eine ventral angebrachte abstützende Platte den Dens adäquat zu stabilisieren. Nach der komplikationslosen Operation zeigt die postoperative Röntgenkontrolle eine Lockerung der eingebrachten proximalen Schraube (Abb. 3).

Diese frühzeitige Implantatlockerung löst Bestürzung im Behandlungsteam aus und man entschließt sich daher in

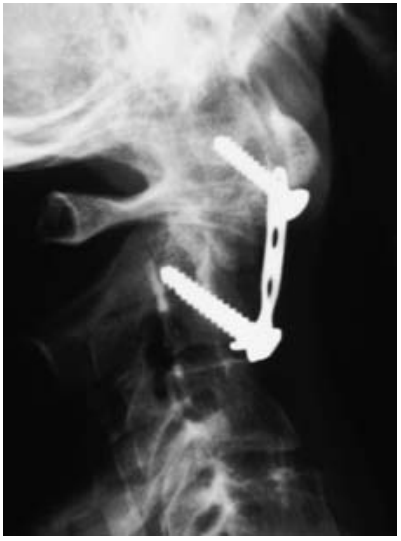

Abb. 3 Ventrale Plattenosteosynthese.

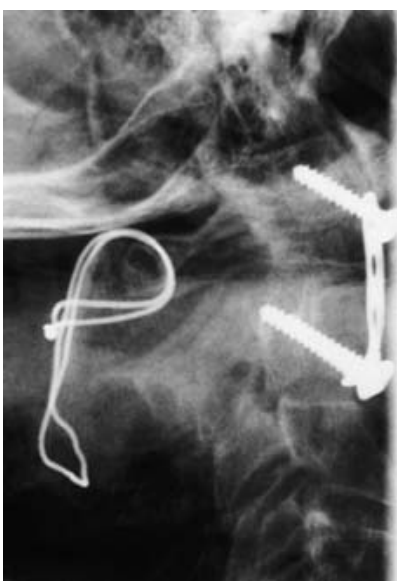

Abb. 4 Zusätzlich dorsale Fusion.

einem 3. Operationsschritt zur Durchführung einer posterioren atlantoaxialen Fusion mit Cerclage (Abb.4).

Zusätzlich wird dem Patienten ein HaloFixateur zur externen Stabilisierung angelegt. Unter dieser maximal durchführbaren Ruhigstellung kommt es bei ansonsten komplikationslosem Heilverlauf zur Auslockerung des Halo-Fixateurs nach 14 Tagen, sodass der Halo-Ring neu platziert am Schädeldach festge- 

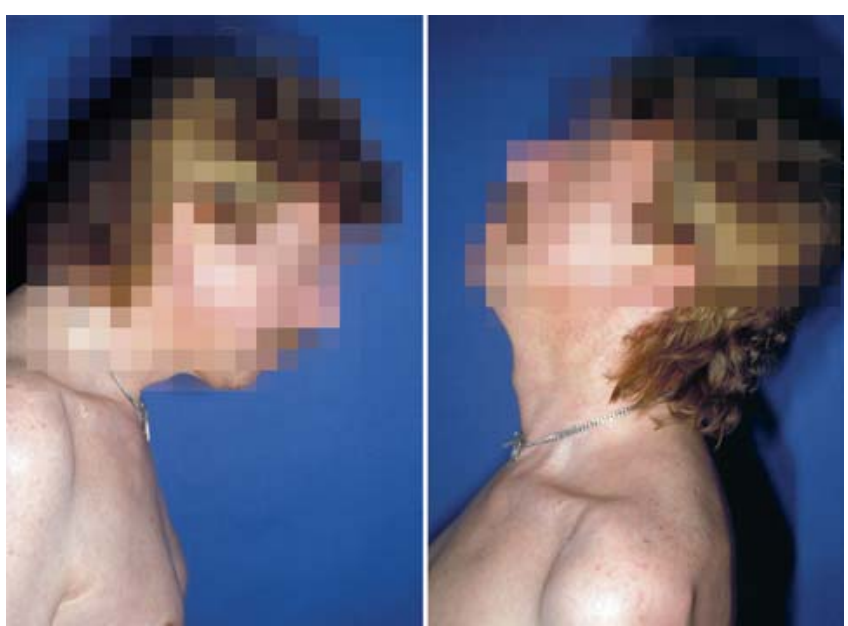

Abb. 5 Funktion

Halswirbelsäule

1 Jahr postoperativ.

schraubt werden muss. In wöchentlichen Kontrollen kommt es unter Nachspannen der Halo-Fixateur-Konstruktion zu einer zeitgerechten knöchernen Durchbauung der Densfraktur. Daraufhin kann der Halo-Fixateur abgenommen werden.

Eine Nachuntersuchung des Patienten nach 1 Jahr zeigt eine mäßiggradige residuelle Bewegungseinschränkung in Flexion und Extension des ansonsten beschwerdefreien Patienten (Abb.5).

Die ausgeprochen mühsamen Behandlungsbemühungen hat der Patient zum Glück ohne schwerwiegende Folgen gut überstanden. Als hauptursächlich für das mehrfache Implantatversagen wird das hochgradige Stottern angesehen, welches zu unkontrollierbaren, erheblichen Krafteinwirkungen im Bereich der Halswirbelsäule bei jedem Wort geführt hat. Diese Komponente wurde eindeutig bei der Behandlung des Patienten unterschätzt.

\section{Empfehlung}

Sprechen Sie mit dem Patienten, bevor Sie ihn operieren und seien Sie bei Stotterern hinsichtlich der Halswirbelsäule besonders vorsichtig!
Fazit

Die Tatsache des ausgeprägten Stotterns (wissenschaftlich als Erkrankung namens Balbuties bekannt) ist nicht ausreichend in die Indikationsstellung zur Behandlung der Densfraktur eingeflossen und lässt die mechanische Unruhe erklären. Die primär technisch insuffiziente Ausführung der Operation im Wochenendnotdienst hat das Versagen der Operationsmethode vorprogrammiert. Letztlich konnte nur durch Stabilisierung mittels „Gürtel und Hosenträger", d. h. kombinierte ventrodorsale Osteosynthese plus Halo-FixateurAnlage das Frakturareal ausreichend ruhig gestellt werden.

Prof. Dr. med. Michael Nerlich Leiter der Abteilung Unfallchirurgie

Abteilung Unfallchirurgie Universitätsklinikum Regensburg Franz-Josef-Strauß-Allee 11 93042 Regensburg

E-Mail: michael.nerlich@ klinik.uni-regensburg.de 